\title{
O que podemos aprender com os Trabalhos de Conclusão de Curso de graduação em Odontologia? Um estudo bibliométrico
}

\author{
Samantha Vieira*; Maiara Kessin Geraldi*; Doris Gomes**; Renata Goulart Castro***; Mirelle \\ Finkler $* * *$ \\ * Graduanda em Odontologia, Universidade Federal de Santa \\ Catarina \\ ** Doutoranda em Odontologia, Universidade Federal de Santa \\ Catarina \\ *** Doutora em Odontologia, Docente, Departamento de Odonto- \\ logia, Universidade Federal de Santa Catarina
}

Recebido em 03/11/2017. Aprovado em 13/03/2018.

\begin{abstract}
RESUMO
Buscou-se analisar a produção científica do curso de graduação em Odontologia da Universidade Federal de Santa Catarina, por meio do levantamento dos Trabalhos de Conclusão de Curso realizados nos primeiros seis anos de implantação do novo currículo. Realizou-se análise bibliométrica dos trabalhos defendidos até 2016 e disponíveis no website da biblioteca universitária. Coletaram-se as variáveis: ano de publicação, áreas de pesquisa, formato da publicação, tipo de trabalho, abordagem, método, objetivo, coleta dos dados, cenários de pesquisa e submissão a Comitês de Ética em Pesquisa. Foi procedida análise descritiva dos 413 trabalhos levantados, sendo em média, 68,83 trabalhos defendidos por ano. Do total, 63,68\% foram categorizadas na área de "especialidades odontológicas", destacando-se a dentística restauradora com 23\%. A área de Saúde Bucal Coletiva e afins representou $13 \%$ e as "ciências básicas" $3 \%$. Um total de $84,75 \%$ dos TCC foram realizados no formato de monografia e 63,20\% como "pesquisa científica". Destes, 92,34\% foram de abordagem quantitativa, $65,90 \%$ com método observacional, $61 \%$ com objetivo descritivo, 70,75\% com dados coletados de forma transversal, 67\% submetidos a Comitês de Ética em Pesquisa, e 44,20\% desenvolvidos em "serviços públicos". Considera-se que o perfil da produção científica ainda segue o modelo tradicional de Odontologia, coerente com sua histórica formação clínico-cirúrgico-tecnicista, a despeito das mudanças preconizadas pelas Diretrizes Curriculares Nacionais. Sugere-se um planejamento desta produção científica voltada a uma formação condizente com as necessidades em saúde bucal da maioria da população brasileira.
\end{abstract}

Descritores: Pesquisa. Educação Superior. Currículo. Bibliometria. Odontologia. 


\section{INTRODUÇÃO}

Os cursos de graduação em Odontologia brasileiros, têm historicamente formado cirurgiões-dentistas (CD) para exercerem a profissão no mercado privado, com um perfil de assistência à saúde curativo e individualizado, associado a forte agregação de investimento tecnológico material e conhecimentos técnicoespecializados. Seguindo o modelo liberalbiomédico de formação, apoia-se na fragmentação de conteúdos e no ensino centrado no professor especialista, distanciando o acadêmico da preparação para o cuidado integral em saúde bucal $(\mathrm{SB})^{1}$. Coerentemente a este modelo hegemônico de formação e atuação profissional, as pesquisas científicas na área da Odontologia caracterizam-se pelo enfoque clínico voltado às especialidades ${ }^{2}$. Uma produção que é recente e oriunda, majoritariamente, das universidades e programas de pós-graduação públicos - que respondem por $70 \%$ de toda a produção científica brasileira ${ }^{3}$ situando nosso país entre os mais produtivos do mundo ${ }^{4}$.

As instituições públicas de ensino superior são constituídas sobre o tripé ensino, pesquisa e extensão, incentivando seus membros a aumentarem cada vez mais sua produção científica, alicerçada nas exigências das agências de avaliação e fomento de pesquisa acadêmica ${ }^{5}$. Entretanto, as pesquisas em odontologia ainda mantém-se voltadas, principalmetne, aos interesses de mercado ${ }^{6}$, em detrimento de maior investimento nos avanços do Sistema Único de Saúde (SUS), consoantes com as necessidades sociais e epidemiológicas de ampla maioria da população do país.

A implantação das Diretrizes Curriculares Nacionais (DCN), em 2002, procurou modificar este histórico processo de produção da ciência e formação na saúde, estimulando a elaboração de projetos pedagógicos voltados às realidades regionais, na busca por uma formação que contemplasse excelência técnica e relevância social $^{7}$. Neste sentido, a flexibilização curricular promovida pelas DCN nos cursos de Odontologia possibilitou modificações na formação acadêmica, significando uma maior liberdade de reorganização dos currículos no sentido da superação do modelo liberalbiomédico $^{8}$. As pesquisas científicas em SB passam a assumir um papel preponderante para construção de um novo perfil de formação e atuação profissional ${ }^{7}$, pautado em novo modelo de atenção à saúde que ressalta as tecnologias relacionais multiprofissionais e interdisciplinares, com ênfase na comunicação entre os atores e na integralidade da atenção, a partir das necessidades epidemiológicas e sociais ${ }^{2}$.

A elaboração de Trabalhos de Conclusão de Curso (TCC) constitui ferramenta importante para o desenvolvimento deste novo profissional crítico e reflexivo ${ }^{9}$, quando a formação tem como objetivo o desenvolvimento de um profissional ativo na construção do seu conhecimento, com excelência técnico-científica e também éticohumanística. Assim, o TCC passa a ser compreendido não como mera tarefa exigida pelo projeto pedagógico dos cursos, mas como conquista fundamental para estimular a criatividade, a educação permanente e a busca dos acadêmicos por conhecimento científico qualificado, a partir da construção de projetos coerentes com os princípios das $\mathrm{DCN}^{10}$. O TCC busca aproximar os acadêmicos do método científico, a partir da confecção de monografia, publicações e apresentações de trabalhos científicos em eventos acadêmicos, para que reconheçam o valor da pesquisa científica no desenvolvimento profissional ${ }^{11}$ e também como compromisso social.

Neste sentido, analisar os TCC realizados nos cursos de graduação em Odontologia possibilita, além de identificar o perfil da 
pesquisa acadêmica, verificar sua contribuição para o delineamento dos rumos da pesquisa científica no meio acadêmico e perceber a consonância dos cursos às orientações das $\mathrm{DCN}^{3,9,12}$. Objetivou-se neste trabalho, analisar a produção científica no curso de graduação em Odontologia da Universidade Federal de Santa Catarina (UFSC) relacionada aos TCC produzidos a partir da implantação de seu último currículo.

\section{MATERIAL E MÉTODOS}

O presente estudo bibliométrico de abordagem quantitativa, transversal, de característica exploratória e descritiva, foi realizado com base nos TCC do curso de graduação em Odontologia da UFSC defendidos entre os anos de 2011 e 2016. Os dados foram levantados em base pública, disponível on line, no site da Biblioteca Universitária (BU) da UFSC, sendo que os títulos dos trabalhos e os nomes de seus autores foram guardados em sigilo, não sendo apresentadas informações diretas que permitam identificá-los.

As variáveis levantadas foram: título do trabalho; ano de publicação; área temática do trabalho, categorizadas em "ciências básicas", "especialidades odontológicas", "Saúde Bucal Coletiva e áreas afins" ou "Odontologia em geral" (esta última categoria incluiu todos os temas que não se enquadravam nas demais categorias); formato da publicação, categorizado como "artigo" ou "monografia"; tipo do trabalho, caracterizado como "revisão bibliográfica", "pesquisa científica", "relato de caso clínico" ou "relato de experiência em atividade de extensão" (estas duas últimas variáveis foram definidas a partir das modalidades permitidas pelo regulamento dos TCC do curso estudado). Os trabalhos categorizados em pesquisa científica foram sub categorizados segundo a abordagem como "quantitativo" ou "qualitativo"; método como "experimental" ou "observacional"; objetivo como "exploratório", "descritivo" ou "analítico"; tipo de dados como "transversal" ou "longitudinal"; e quanto submissão ao Comitê de Ética em Pesquisa "sim" ou "não". Além destas, foi levantado ainda o "cenário", categorizado como: "serviços públicos", "laboratórios de pesquisa", "literatura", "serviços público-privados", "serviços privados" ou "documentos públicos".

Para coleta de dados foi elaborada uma planilha de dados, além de uma calibração teórica prévia, a partir da análise dos dados de alguns TCC pelas pesquisadoras. Após a coleta, as mesmas realizaram uma reunião para consenso dos dados obtidos, e os trabalhos com dúvidas foram novamente discutidos para a categorização final e prosseguimento da análise exploratória e descritiva dos dados.

\section{RESULTADOS}

Foram analisados 413 trabalhos disponíveis no site da BU da UFSC. Os dados coletados foram sintetizados na tabela 1 .

A média de trabalhos publicados por ano foi $69( \pm 24)$, sendo 2011 o ano com menor número de trabalhos (24 TCC ou 5,81\%), ano em que apenas uma turma apresentou seus trabalhos, e 2014 o ano com o maior número (103 TCC ou 24,94\%).

A área de "especialidades odontológicas" foi a mais frequente, com $263(63,68 \%)$ trabalhos, seguida pela "Odontologia em geral" com 82 (19,85\%), pela "Saúde Coletiva e áreas afins" com $54(13,08 \%)$ e pelas "ciências básicas" com 14 (3,39\%).

Dos trabalhos levantados, 63 (15,25\%) foram elaborados em formato de artigo científico e $350(84,75 \%)$ em formato de monografia.

Quanto ao tipo de trabalho realizado, 261 $(63,20 \%)$ foram considerados "pesquisas científicas", $139(33,66 \%)$ "revisões bibliográficas", $12(2,91 \%)$ "relatos de caso clínico" e $1(0,24 \%)$ "relato de experiência em atividade de extensão". 
Tabela 1. Distribuição dos trabalhos de conclusão do curso de odontologia da UFSC publicados de 2011 a 2016 , pelos dados coletados, Florianópolis/SC

\begin{tabular}{|c|c|c|}
\hline Variável & $\mathbf{n}$ & $\%$ \\
\hline \multicolumn{3}{|l|}{ Ano de publicação } \\
\hline 2016 & 71 & $17,19 \%$ \\
\hline 2015 & 80 & $19,37 \%$ \\
\hline 2014 & 103 & $24,94 \%$ \\
\hline 2013 & 65 & $15,74 \%$ \\
\hline 2012 & 70 & $16,95 \%$ \\
\hline 2011 & 24 & $5,81 \%$ \\
\hline Total & 413 & $100,00 \%$ \\
\hline \multicolumn{3}{|l|}{ Áreas de pesquisa } \\
\hline Especialidades odontológicas & 263 & $63,68 \%$ \\
\hline Odontologia em geral & 82 & $19,85 \%$ \\
\hline Saúde bucal coletiva e áreas afins & 54 & $13,08 \%$ \\
\hline Ciências básicas & 14 & $3,39 \%$ \\
\hline Total & 413 & $100,00 \%$ \\
\hline \multicolumn{3}{|l|}{ Formato da publicação } \\
\hline Monografia & 350 & $84,75 \%$ \\
\hline Artigo & 63 & $15,25 \%$ \\
\hline Total & 413 & $100,00 \%$ \\
\hline \multicolumn{3}{|l|}{ Tipo do trabalho } \\
\hline Pesquisa científica & 261 & $63,20 \%$ \\
\hline Revisão bibliográfica & 139 & $33,66 \%$ \\
\hline Relato de caso clínico & 12 & $2,91 \%$ \\
\hline Relato de experiência em atividade de extensão & 1 & $0,24 \%$ \\
\hline Total & 413 & $100,00 \%$ \\
\hline \multicolumn{3}{|l|}{ Abordagem } \\
\hline Quantitativa & 241 & $92,34 \%$ \\
\hline Qualitativa & 20 & $7,66 \%$ \\
\hline Total & 261 & $100,00 \%$ \\
\hline \multicolumn{3}{|l|}{ Método } \\
\hline Observacional & 171 & $65,51 \%$ \\
\hline Experimental & 90 & $34,49 \%$ \\
\hline Total & 261 & $100,00 \%$ \\
\hline \multicolumn{3}{|l|}{ Objetivo } \\
\hline Descritivo & 159 & $60,92 \%$ \\
\hline Analítico & 100 & $38,31 \%$ \\
\hline Exploratório & 2 & $0,77 \%$ \\
\hline Total & 261 & $100,00 \%$ \\
\hline \multicolumn{3}{|l|}{ Dados } \\
\hline Transversal & 177 & $67,82 \%$ \\
\hline Longitudinal & 84 & $32,18 \%$ \\
\hline Total & 261 & $100,00 \%$ \\
\hline \multicolumn{3}{|l|}{ Submissão ao Comitê de Ética em Pesquisa } \\
\hline Sim & 205 & $33,00 \%$ \\
\hline Não & 101 & $67,00 \%$ \\
\hline Total & 306 & $100,00 \%$ \\
\hline \multicolumn{3}{|l|}{ Cenário } \\
\hline Serviços públicos & 130 & $44,22 \%$ \\
\hline Laboratórios de pesquisa & 69 & $23,47 \%$ \\
\hline Literatura & 39 & $13,27 \%$ \\
\hline Serviços público-privados & 28 & $9,52 \%$ \\
\hline Serviços privados & 14 & $4,76 \%$ \\
\hline Documentos públicos & 14 & $4,76 \%$ \\
\hline Total & 294 & $100,00 \%$ \\
\hline
\end{tabular}


Dos 261 trabalhos categorizados como pesquisa científica, 241 (92,34\%) foram de abordagem quantitativa e $20 \quad(7,66 \%)$ de abordagem qualitativa. Quanto ao método empregado, $171(65,51 \%)$ foram considerados observacionais e $90(34,49 \%)$ experimentais. Em relação ao objetivo, o mais frequente foi o estudo descritivo com 159 (60,92\%), seguido do analítico com 100 (38,31\%), e do exploratório com $2(0,77 \%)$. Os trabalhos com dados transversais foram os mais frequentes, com 177 $(67,81 \%)$, seguidos por $84(32,18 \%)$ TCC com dados longitudinais.

Dos 306 trabalhos totalizados como "pesquisa científica" e "relato de caso clínico" (aqueles que demandam avaliação ética, sempre que envolvem participação de seres humanos ou animais), 205 (67\%) foram submetidos a Comitês de Ética em Pesquisa com Seres Humanos (CEP) ou a Comissões de Ética no Uso de Animais (CEUA), o que corresponde à soma das pesquisas realizadas em "serviços públicos", "privado", "público-privados", além dos relatos de caso clínico. Destes 205 TCC submetidos aos CEP, 63 (30,7\%), foram elaborados em formato de artigo.

$\mathrm{O}$ cenário mais frequente foi $\mathrm{o}$ dos "serviços públicos" com 130 (44,22\%), seguidos por "laboratórios de pesquisa" com 69 (23,47\%), "literatura" com 39 (13,27\%), "serviços públicoprivados com $28(9,52 \%)$, "serviços privados" com $14(4,76 \%)$ e "documentos públicos" com $14(4,76 \%)$.

\section{DISCUSSÃO}

A partir da mudança curricular ocorrida no curso de Odontologia da UFSC e a consequente obrigatoriedade do TCC para sua conclusão, todos os estudantes tiveram a possibilidade de maior contato com a construção do conhecimento científico, passando a pesquisa a ser valorizada como parte do processo educativo e reflexivo, na graduação e de forma permanente na vida profissional. A variabilidade encontrada no número de trabalhos publicados por ano reflete as sinuosidades dos caminhos percorridos pelos estudantes ao longo do curso, bem como a ampliação de vagas a partir de 2009 (de 90 para 100 vagas anuais).

Os resultados apontam que a valorização de diferentes áreas e especialidades no curso de graduação pode estar relacionada ao interesse e envolvimento direto dos estudantes com as disciplinas curriculares, com o respeito e a admiração pelos professores ou disciplinas e, ainda, pela presença e tradição da pós-graduação na instituição, historicamente alinhada aos interesses de mercado. Assim, a escolha da temática de pesquisa e de orientadores para o TCC parece revelar a maior ou menor influência do currículo oculto sobre os estudantes e suas preferências $^{8}$, especialmente observada em relação às áreas técnicas e clínicas, coadunando resultados de outros estudos ${ }^{9,10,12}$. Contexto que facilita a compreensão sobre a predominância da área temática "especialidades odontológicas", observada, também, no estudo sobre TCC na Universidade Federal de Pelotas (UFPel) ${ }^{9}$, com maior concentração de TCC em Dentística; na Escola Bahiana de Medicina e Saúde Pública $(\mathrm{EBMSP})^{12}$, com maioria dos trabalhos na Estomatologia; e na Universidade Federal do Rio Grande do Norte (UFRN), em Patologia Bucal ${ }^{10}$. Além disto, podem ser considerados fatores que interferem nas escolhas dos estudantes a presença de pós-graduandos em equipes de pesquisa e como co-orientadores de TCC; a possibilidade de bolsas de iniciação científica por meio de professores credenciados a programas de pós-graduação; e a maior valorização das áreas que dela participam. Leite et al. ${ }^{10}$ alertam que também pode se constituir um viés de interferência nestas escolhas, a oferta tardia de determinadas áreas no curso, após o 
início da elaboração do TCC, visto que as áreas mais pesquisadas são estudadas nos períodos iniciais - viés não confirmado neste trabalho.

A procura pela área "Saúde Bucal Coletiva e áreas afins" (13,08\%) como temática para desenvolvimento de TCC pode ser considerada baixa, em especial, pela existência de um eixo transversal de Saúde Coletiva no curso da UFSC e, sobretudo, pela centralidade da área em relação à relevância social da pesquisa científica desenvolvida como TCC. A conformação histórica do modelo de ensino (flexneriano), de profissão (liberal) e de ciência (biologicista), enraizados na super-especialização voltada à atividade clínica procedimento-centrada e formatada por interesses de mercado, mantém-se hegemônica na sua relação com a tipologia das pesquisas desenvolvidas. No mesmo sentido, a prática clínica tradicional organizada em torno do hospital-escola, apesar de sua inserção como serviço do SUS, ainda resiste às mudanças de transversalidade da saúde coletiva implementadas no novo currículo, mantendo-se a tradição da clínica fragmentada por especialidades. Estes são elementos que podem explicar a baixa procura pela realização de pesquisas nesta área.

Ainda assim, alguns pesquisadores indicam progressos que acenam para uma nova realidade acadêmica, na qual o interesse pelo social vem se consolidando como objeto de estudo $^{2}$. Neste sentido, caberia questionar qual a relação entre as pesquisas desenvolvidas na odontologia da UFSC e a Agenda Nacional de Prioridades em Pesquisas na Saúde (ANPPS), tendo em vista a insuficiente interdisciplinaridade entre as disciplinas do curso e as da área de Saúde Bucal Coletiva ${ }^{13}$, atestada pela ausência de um planejamento estratégico do curso que priorize a execução de pesquisas voltadas ao melhoramento da atenção à saúde à maioria da população. Como consequência destas escolhas, mantém-se a pesquisa em Odontologia, majoritariamente, nos moldes tradicionais.

Apesar dos TCC da UFSC apontarem forte produção de novos conhecimentos, apenas $15 \%$ foram desenvolvidos em formato de artigo científico. Como este permitiria uma maior rapidez na divulgação do conhecimento gerado, questiona-se acerca da intencionalidade da publicação dos conhecimentos e técnicas desenvolvidos nos TCC em periódicos, bem como sua apresentação em eventos científicos. O curto tempo para o planejamento e execução de um TCC pode resultar numa perspectiva diminuída de publicação científica, principalmente considerando-se a dificuldade atual na publicação de artigos científicos, não se completando o processo de produçãodivulgação-apropriação de conhecimentos ${ }^{2}$. De acordo com o estudo realizado na UFPel, apenas 9\% dos TCC foram publicados em periódicos científicos $^{9}$, o que pode representar sério problema ao processo de apropriação social do conhecimento produzido. Esta preocupação aponta para que os TCC não devam representar apenas um cumprimento de exigência curricular, mas sim seu papel científico e social, proporcionando respostas efetivas e acessíveis aos problemas candentes da sociedade que os financia. Em alguns cursos, como $\mathrm{o}$ de Odontologia da EBMSP ${ }^{12}$, o TCC obrigatoriamente é redigido na forma de artigo científico, percebendo-o como um importante resultado em termos de produção, já que a publicação em periódicos, livros ou anais de congressos, amplia a difusão dos conhecimentos gerados ${ }^{5}$.

Observou-se predominância de TCC do tipo "pesquisa científica" $(63,20 \%)$ seguidos do "revisão bibliográfica" $(33,66 \%)$ e do "relato de caso clínico" (2,91\%). No estudo realizado na EBMSP, apenas $36 \%$ dos trabalhos 
correspondiam a "pesquisa", 29\% a "revisão de literatura" e $35 \%$ a "relato de caso"12. Já na UFPel, esses dados foram respectivamente $51 \%$, $21 \%$ e $27 \%{ }^{9}$. Esta maioria de trabalhos no tipo "pesquisa científica" produzidos na UFSC é um aspecto positivo, visto seu potencial de produção de novos conhecimentos científicos.

Dentre os trabalhos classificados como do tipo "pesquisa científica", a maioria $(92,34 \%)$ possuía abordagem "quantitativa", o que denota a hegemonia da corrente de pensamento positivista/neo positivista e cartesiana na construção do conhecimento no curso. As pesquisas qualitativas em saúde, apesar de seu amplo potencial para responder a problemas não traduzíveis em números, não solucionados com abordagens epidemiológicas, experimentais e outros desenhos de base quantitativa, oferecendo a possibilidade de produção de conhecimento mais aprofundado sobre fenômenos subjetivos humanos presentes nos processos de saúdedoença e relacionais da realidade social, ainda são preteridos aos métodos quantificáveis. Uma abordagem também dificultada pelo pouco espaço que os periódicos científicos destinam a pesquisas desta natureza, influenciando na definição do projeto ${ }^{14}$.

Os trabalhos com desenvolvimento de pesquisas "observacionais" representaram praticamente o dobro dos trabalhos "experimentais", com 65,90\% e 34,10\%, respectivamente. Provavelmente, porque a realização de estudos experimentais demanda um tempo maior, aumentando também a chance de contingências e requerendo um início mais precoce do estudante de graduação aos projetos de pesquisa, antes mesmo que os conteúdos de metodologia da pesquisa e dos TCC comecem a ser trabalhados no curso.

Neste sentido, sugere-se a criação de mecanismos de incentivo e controle para tornar homogêneo e constante o processo de pesquisa ao longo da graduação em odontologia ${ }^{15}$, percebendo as produções científicas como incentivadoras da autonomia, de sua habilidade e iniciativa de pensar a realidade e de, eventualmente, nela intervir de maneira crítica e reflexiva ${ }^{16}$. Ainda, a possibilidade de ingresso antecipado em grupos de pesquisa poderia estimular o contato e a participação do estudante com projetos, bem como poderia proporcionar um tempo maior para o desenvolvimento do trabalho, já que os grupos possuem uma organização independente do tempo da graduação, além de trajetória e experiência nas áreas temáticas a que se propõem.

A maioria dos TCC de "pesquisa científica" analisados apresentou o objetivo de "descrever" dados levantados de forma "transversal". Outros estudos constataram resultados semelhantes ${ }^{3,6,10}$. Este tipo de pesquisa possui um baixo custo operacional, simplicidade analítica, alto potencial descritivo e rapidez na coleta de dados, além da facilidade em representar aspectos de uma população ${ }^{10}$. Levando em consideração o tempo de três semestres para a conclusão do TCC conforme o currículo do curso analisado, torna-se uma boa alternativa para pesquisadores iniciantes. Por outro lado, resultam, em sua maioria, em trabalhos de pequeno impacto científico, com maior dificuldade de publicação.

Em relação aos "cenários", a maior parte dos TCC foi realizada em "serviços públicos". A variedade de "cenários de pesquisa" encontrada em outros "serviços púbicos" além da UFSC, aponta para uma diversidade de espaços que incluem Hospitais, Unidades Básicas de Saúde e Centro de Especialidades Odontológicas, não ficando restritos os TCC, portanto, somente a pesquisas realizadas na própria instituição ${ }^{10}$. A viabilidade na realização de pesquisas extramuros possibilita a construção de um olhar mais crítico por parte do futuro profissional, para 
além da clínica-escola. Neste estudo, fortalece-se a ideia de que a iniciação científica no ambiente do SUS é fundamental para que o acadêmico vivencie o que lhe é exposto nas aulas teóricas, gerando um diálogo crítico e desenvolvendo sua autonomia no processo de aprendizagem ${ }^{17}$. É necessário o contato do graduando com o SUS também no momento da pesquisa, tendo em vista o distanciamento das vivências nas clínicas da instituição com a vivência prática na rede de atendimento do sistema de saúde ${ }^{18}$. Entretanto, o predomínio do local de estudo nos "serviços públicos" explicado pela facilidade de acesso, disponibilidade de recursos e a participação dos professores orientadores na pesquisa, ainda aponta uma predominância dos espaços intramuros, visto que dentre os locais predominantes, estão a Clínica Odontológica e o Hospital Universitário, ambos da UFSC.

A maioria dos TCC de "pesquisa científica" e "relato de caso clínico" foram submetidos a CEP, corroborando resultado de outro estudo que aponta a viabilidade de realização de pesquisas que envolvem seres humanos também na graduação, apesar de um menor tempo disponível para as pesquisas e o despreparo científico dos acadêmicos, quando comparado às realizadas em nível de pósgraduação $^{10}$. Tais dados ratificam um tempo exímio de aprovação dos projetos de pesquisa submetidos aos CEP.

Além disso as pesquisas aprovadas pelo CEP, elaboradas em formato de artigo, estão acima da média geral total, indicando que apesar da predominância do formato de monografia, há maior chance de publicação dos resultados. Seguindo a Resolução CNS 466/201212, o pesquisador $\mathrm{e}$ as instituições possuem o compromisso de encaminhar os resultados para publicação ou, então, apresentar uma justificativa para sua não publicação, observada a relevância social da publicação dos resultados, sobretudo daqueles que envolvam seres humanos. Considera-se positivo o fato de estudantes realizarem pesquisas com seres humanos e não estarem restritos a pesquisas no campo laboratorial, sugerindo avanços no que diz respeito à tradicional ênfase nas pesquisas de materiais ${ }^{6}$.

Sendo a construção de conhecimento científico uma atividade central na instituição universitária contemporânea, com resultados que se refletem nas esferas científica, social e econômica $^{19}$, o perfil dos TCC realizados na graduação em odontologia da UFSC parece encontrar-se, ainda, fragmentado e desarticulado das necessidades sociais e epidemiológicas da sociedade na qual está inserido.

\section{CONCLUSÃO}

O perfil da produção científica identificado neste estudo corresponde predominantemente a trabalhos na área de "especialidades odontológicas", no formato de "monografia", do tipo "pesquisa científica", desenvolvidos em "serviços públicos", com o aval dos comitês de ética em pesquisa. Os trabalhos de "pesquisa científica", por sua vez, apresentaram majoritariamente abordagem "quantitativa", método "observacional", com objetivo "descritivo" e dados coletados de forma "transversal". Desta forma, percebe-se ainda, uma lacuna em relação ao processo de integração entre as especialidades odontológicas, a formação generalista e a Saúde Bucal Coletiva, pela verificação do enfoque dos trabalhos nas áreas de "especialidades odontológicas". O perfil dos TCC referenda um modelo profissional ainda fragmentado e desarticulado da sociedade e do sistema de saúde ao qual deveria estar inserido.

Frente a esta realidade, ressalta-se a importância da construção de projetos de pesquisas à luz das DCN e, especialmente, o investimento em estudos científicos 
comprometidos com os avanços na atenção à saúde bucal praticados no SUS. Neste sentido, aponta-se para a necessidade do desenvolvimento de trabalhos integrando as áreas técnicas e clínicas com a Saúde Bucal Coletiva, a partir de um planejamento estratégico institucional que leve em consideração a ANPPS.

Sugere-se, ainda, ampliar a possibilidade de incorporação dos acadêmicos a grupos de pesquisa já no início da graduação, otimizando suas oportunidades de realizar pesquisa científica, bem como $\mathrm{o}$ fomento à responsabilidade ética de professores e acadêmicos quanto à eleição das temáticasobjeto de pesquisa nos TCC e à publicização de seus resultados, de forma a promover a eticidade da prática investigativa.

\section{ABSTRACT \\ What can we learn from Undergratuate Final Project in Dental Schools? A bibliometric study}

The aim of this study was to analyze the scientific production of the undergraduate course in Dentistry at UFSC, by means of a survey of the Final Projects carried out in the first six years of implementation of the new curriculum. A bibliometric analysis of the works defended until 2016 and available on the university library website. The following data were collected: year of publication, research areas, publication format, type of work, approach, method, objective, data collection, research scenarios and submission to Research Ethics Committees. A descriptive analysis was carried out of the 413 papers surveyed, with an average of 68.83 works defended per year. Of the total, $63.68 \%$ were categorized in the area of "Dental Specialties", highlighting restorative dentistry with $23 \%$. The area of Oral and Collective Health represented $13 \%$ and basic sciences $3 \%$. A total of $84.75 \%$ of the Final Reports were performed in the monograph format and $63.20 \%$ as "Scientific Research". Of these, $92.34 \%$ were of a quantitative approach, $65.90 \%$ with observational method, $61 \%$ with descriptive purpose, $70.75 \%$ with cross-sectional data, 44.20\% developed in "Public Services" and 67\% submitted to Research Ethics Committees. It is considered that the profile of scientific production still follows the traditional model of Dentistry, consistent with its historical clinicalsurgical-technicist formation, in spite of the changes advocated by the National Curricular Guidelines. It is suggested a planning of this scientific production aimed at a training compatible with the oral health needs of the majority of the Brazilian population.

Descriptors: Research. Education, Higher. Curriculum. Bibliometrics. Dentistry.

\section{REFERÊNCIAS}

1. Toassi RFC, Souza JM, Baumgarten A, Rosing CK. Avaliação curricular na educação superior em odontologia: discutindo as mudanças curriculares na formação em saúde no Brasil. Rev ABENO. 2012; 12(2):170-7.

2. Dias AA, Narvai PC, Rêgo DM. Tendências da produção científica em odontologia no Brasil. Rev Panam Salud Pública. 2008; 24(1):54-60.

3. Scariot R, Stadler AF, Assunção CM, Pintarelli TP, Ferreira FM. A map of Brazilian dental research in the last decade. Braz Oral Res. 2011; 25(3):197-204.

4. Xavier AFC, Silva ALÓ, Cavalcanti AL. Análise da produção científica em Odontologia no nordeste brasileiro com base em um congresso odontológico. Arq Odontol. 2011; 47(3):127-34.

5. Silva MR, Hayashi CAM, Hayashi MCPI. Análise bibliométrica e cientométrica: desafios para especialistas que atuam no campo. Rev Ci Inf Doc. 2011; 2(1):110-29.

6. Finkler M, Calvo MC, Caetano JC, Ramos FRS. Um novo olhar bioético sobre as pesquisas odontológicas brasileiras. Ciênc Saúde Colet. 2009; 14(4):1205-14. 
7. Brasil. Ministério da Educação. Conselho Nacional de Educação. Câmara de Educação Superior. Resolução CNE-CES n.3. Diretrizes Curriculares Nacionais do Curso de Graduação em odontologia. Diário Oficial da União, Brasília, 4.mar. 2002. Seção 1, p.10. [Acesso em: 17 out. 2017]. Disponível em: http://portal.mec.gov.br/cne/ arquivos/pdf/CES032002.pdf.

8. Finkler M, Caetano JC, Ramos FRS. Modelos, mercados e poder: elementos do currículo oculto que se revelam na formação em odontologia. Trab Educ Saúde. 2014; 12(2):343-61.

9. Chisini LA, Silva HG, Nôbrega KHS, Conde MCM, Corrêa MB, Demarco FF. Análise descritiva dos trabalhos de conclusão de curso da Faculdade de Odontologia, UFPel. Rev ABENO. 2017; 17(1):8-15.

10. Leite B, Menêzes T, Noro L. Análise bibliométrica de Trabalhos de Conclusão de um Curso de Odontologia no Nordeste brasileiro. Rev ABENO. 2015; 15(3):16-25.

11. Dias CM. O trabalho de conclusão de curso na percepção dos alunos de pedagogia da UEL [Trabalho de Conclusão de Curso]. Curso de Graduação em Pedagogia, UEL; 2011. [Acesso em 17 out. 2017]. Disponível em: http://www.uel.br/ceca/pedagogia/pages/ arquivos/CILENE\%20MARCONDES\%20 DIAS.pdf.

12. Costa RM, Vieira RS, Cavalcanti AN, Tunes UR, Ribeiro EP, Oliveira, VMB. Perfil da pesquisa acadêmica odontológica da EBMSB. Rev ABENO. 2015; 15(1):70-6.

13. Gomes D, Agnoletto IG, Souza ML, Spiger V, Jakymiu JRG, Fugii EC et al. A produção científica da Odontologia e a Agenda Nacional de Prioridades de Pesquisa em Saúde. Rev ABENO. 2017; 17(2):11-21.
14. Taquette SR, Villela WV. Balizas do conhecimento: análise das instruções aos autores das revistas brasileiras da área de saúde. Ciênc Saúde Colet. 2017; 22(1):7-13.

15. Gabriel M, Tanaka EE. Formação profissional em Odontologia: Percepção discente da interação curricular. Rev ABENO. 2011; 11(1):19-22.

16. Santos BRM. Perfil e expectativas dos ingressantes da Faculdade de Odontologia da USP: uma visão integrada com as diretrizes curriculares nacionais e o sistema único de saúde. Rev ABENO. 2015;15(1): 28-37.

17. Junior MFS, Assis RIF, Sousa HA, Miclos PV, Gomes MJ. Iniciação científica: percepção do interesse de acadêmicos de odontologia de uma universidade brasileira. Saúde Soc. 2014; 23(1):325-35.

18. Cunha MR, Fonsêca GS, Araujo ME, Zilbovicius C. A iniciação científica como estratégia pedagógica para integrar ensino e serviço no SUS. Rev ABENO. 2016; 16(2):33-44.

19. Menêzes TB, Borges LSB, Santos LM, Noro LRA. Análise bibliométrica de trabalhos de conclusão de curso de Nutrição do Rio Grande do Norte. Rev Nutr. 2016; 29(1):139-49.

\section{Correspondência para:}

Doris Gomes

e-mail: dorisgomesodonto@gmail.com

Rod. Rafael da Rocha Pires, 3913

88051-001 Florianópolis/SC 